\title{
A numerical test of stress correlations in fluctuating hydrodynamics
}

\author{
Michael Schindler \\ Laboratoire PCT, UMR "Gulliver" CNRS-ESPCI 7083, 10 rue Vauquelin, 75231 Paris Cedex 05
}

\begin{abstract}
The correlations of the fluctuating stress tensor are calculated in an equilibrium molecular-dynamics simulation of a Lennard-Jones liquid. We define a coarse-grained local stress tensor which can be calculated numerically and which allows for the first time to determine the stress correlation function both in time and in space. Our findings corroborate the assumptions made in fluctuating hydrodynamics as long as the liquid is isotropic, that is in bulk. In the vicinity of a rigid plate, however, the isotropy is restricted, and major modifications must be done with respect to the usual theory. Among these are the appearance of five different viscosities instead of two and a non-trivial dependence of the distance from the wall. We determine these viscosities from the simulation data and find that their values are very different from the bulk values. We further find much longer relaxation times of the stress correlations than in bulk.
\end{abstract}

(

\section{Introduction}

The theory of fluctuating hydrodynamics starts with classical hydrodynamics, which describes the average motion of a fluid and which is formulated in terms of smooth tensor fields, and enriches it by additive fluctuations. It can be understood as the attempt to bridge the gap between microfluidics and nanofluidics from the large-scales side. The central question is of course, what properties the fluctuations must have in order to correctly represent the microscopic states of the liquid in a given problem? The standard approach [1, 2] is to split the full velocity and stress tensor into a macroscopic (denoted by an overbar in the following) and into fluctuating parts (denoted by a tilde). The linearised governing equations take then the form of a two-scale process, with the standard compressible Navier-Stokes equations for the macroscopic velocity $\overline{\mathbf{v}}(\mathbf{x}, t)$ and the corresponding Newtonian stress tensor $\bar{\sigma}_{i j}(\mathbf{x}, t)$,

$$
\bar{\sigma}_{i j}=-\delta_{i j} \bar{p}(\mathbf{x}, t)+\eta\left(\partial_{i} \bar{v}_{j}+\partial_{j} \bar{v}_{i}\right)+\left(\lambda-\frac{2}{3} \eta\right) \delta_{i j} \operatorname{div} \overline{\mathbf{v}} .
$$

Here and in the following, $\eta$ denotes the shear viscosity of the fluid and $\lambda$ its volume viscosity. As we will be concerned only about equilibrium situations, the macroscopic equations reduce to

$$
\bar{\rho}(\mathbf{x}, t)=\rho_{0}, \quad \bar{p}(\mathbf{x}, t)=p_{0}, \quad \overline{\mathbf{v}}(\mathbf{x}, t)=\mathbf{0},
$$

with constants $\rho_{0}$ and $p_{0}$. The fluctuating fields satisfy a linearised Navier-Stokes equation with additional random noise $s_{i j}$,

$$
\begin{aligned}
\rho_{0} \partial_{t} \tilde{\mathbf{v}} & =\operatorname{div}(\tilde{\sigma}+s), \\
\partial_{t} \tilde{\rho} & =-\rho_{0} \operatorname{div} \tilde{\mathbf{v}} .
\end{aligned}
$$

Email address: michael.schindler@espci.fr (Michael Schindler)
The stress tensor in equation (3) is again split into two parts, the first of which satisfies the same Newtonian definition (1) as the macroscopic stress tensor, only in terms of a fluctuating velocity $\tilde{\mathbf{v}}(\mathbf{x}, t)$ and of a fluctuating pressure $\tilde{p}(\mathbf{x}, t)$. The second contribution is an uncorrelated fluctuating stress tensor $s_{i j}(\mathbf{x}, t)$. The tensor $\tilde{\sigma}_{i j}$ is meant as a coarse-grained stress which is fluctuating, but not as randomly as $s_{i j}$, see for example the book by Kubo et al. [3] for coarse-graining concepts, and Ref. [4] for substitute Markov processes. The same two-step separation has been used by Hauge and Martin-Löf [5], Chow and Hermans 6], but without commenting on why it is necessary. Equation (3) has the structure of a Langevin equation, with $s_{i j}$ the random driving. This random stress tensor vanishes on average, $\left\langle s_{i j}(\mathbf{x}, t)\right\rangle=0$, and it has to satisfy the fluctuations-dissipation theorem of the second kind. It is thus uncorrelated in space and in time,

$$
\left\langle s_{i j}(\mathbf{x}, t) s_{k l}(\mathbf{y}, s)\right\rangle=2 k T A_{i j k l} \delta(\mathbf{x}-\mathbf{y}) \delta(t-s) .
$$

Here, $\langle\cdot\rangle$ denotes the average over different realisations, or a time average; $k$ is Boltzmann's constant, and $T$ the temperature of the system. The correlator is further assumed to be isotropic, which fixes the fourth-rank tensor $A_{i j k l}$ to 2 ]

$$
A_{i j k l}=\eta\left[\delta_{i k} \delta_{j l}+\delta_{i l} \delta_{j k}\right]+\left(\lambda-\frac{2}{3} \eta\right) \delta_{i j} \delta_{k l} .
$$

This special form of $A_{i j k l}$ follows from the fact that it must be isotropic, thus invariant under any rotation of the coordinate system. It is therefore a linear combination of the only three isotropic tensors of rank four, which are $\delta_{i j} \delta_{k l}, \delta_{i k} \delta_{j l}$, and $\delta_{i l} \delta_{j k}$ [7]. The factors of proportionality are found to be the three viscosities of a fluid [8]. The additional symmetry of the stress tensor, leading to $A_{i j k l}=A_{j i k l}=A_{i j l k}$ eliminates the rotational viscosity. 
In the vicinity of a rigid boundary, however, isotropy is not granted in the same way as in the bulk. Instead, the stress correlator should reflect the fact that the normal direction is different from the two tangential directions. In this paper, we therefore ask which tensorial form the correlator will adopt when evaluated close to a rigid wall. In order to start with the most simple situation, we regard the stress correlator in thermodynamic equilibrium.

We must further ask, what "close" means, that is up to which distance from the wall the different symmetry is sensed by the fluctuating part of the stress tensor. Near the boundary, we thus question also the spatial delta function in Eq. (5). The problem with the spatial delta function becomes apparent when applying fluctuating hydrodynamics for example to the Brownian motion of a spherical particle. This has been done in the literature on the autocorrelation of a Brownian particle [5, 6]. If we naively integrate the random stress tensor over the particle in order to obtain the random force, and then construct the autocorrelation of that force, the result would always be infinite. The reason is that we then integrate the threedimensional delta function from Eq. (5) only over the twodimensional surface of the particle. The third dimension thus remains a delta function, evaluated at zero.

The spatial delta function must be questioned also from a less formal point of view: The standard argument for the absence of spatial correlations is based on thermodynamics 11: The fluid volume is cut into several small but finite portions $\Delta V$. In each of these volumes, the entropy production is calculated as the volume-integral of the tensorial reduction of the stress with the rate-of-strain. The fluctuations of the stress tensor is then taken from the variations of the entropy production around the equilibrium state. As this integral does not contain any surface contribution, the fluctuations of two stress tensors attributed to two such boxes are independent of each other. This property is then kept even in the limit of infinitesimal box size. It appears as if there should be a lower spatial bound to the validity of this argument, namely when the volume size become of the order of the distance between the molecules of the liquid. As thermodynamic arguments are used, the thermodynamic limit of many interacting molecules is assumed to be done before the box size can be sent to zero. It appears thus as a reasonable question to ask, down to which spatial scale does the delta function yield a good description? It will turn out in the following, that it works amazingly well: In bulk fluid, the delta function holds down to distances smaller than the intermolecular spacing.

It is the aim of this paper to test the validity of Eqs. (5) and (6), describing stress correlations - in particular, to find deviations in the vicinity of a rigid surface. In order to do so, we start from a fully microscopic simulation of a liquid and extract the stress tensor using coarsegraining. As the simulation is done at thermal equilibrium, the macroscopic fluid velocity $\overline{\mathbf{v}}$ vanishes, leaving only the thermodynamic pressure in the macroscopic stress tensor $\bar{\sigma}_{i j}=-p_{0} \delta_{i j}$. We correlate the coarse-grained stress tensor at different positions and at different times in order to verify both the isotropy in Eq. (6) and the deltafunctions in Eq. (5). This will be done both near a rigid wall and in bulk fluid.

The precise form of the stress tensor correlations is potentially important for all fluidic systems including boundaries. In microfluidic applications, and even more in nanofluidic ones, the role of the boundaries is essential [9, 10, 11, 12. In nanofluidics, the presence of boundaries even introduces different time scales in the relaxation dynamics [13. For example, Brownian motion of a particle immersed in a viscous fluid, which is one of the most classical systems both in the hydrodynamic and in the stochastic community, is in most cases a microfluidic problemdepending on the size of the particle. Both for large and for small particles, the velocity fluctuations are known to exhibit long-time tails in their autocorrelation-function. The theoretical explanations of this phenomenon, as far as they consider both the particle and the fluid, assume the isotropic form of Eqs. (5) and (6) for the fluctuating part of the stress tensor - even when this stress tensor is integrated over the surface of the particle to yield the total fluctuating force acting on it [5, 6]. The investigation in the present paper is one of two necessary steps to render the theoretical description of the long-time tails more consistent. We will find below that it is not the usual viscosity which occurs in the formula of the force autocorrelation, but a viscosity which is a property of the fluid and the structuring effect of the rigid particle itself. The other step will be published in Ref. [14].

We will further find that the inclusion of boundaries makes it necessary to pass from homogeneous viscosities (which are defined in the limit of homogeneous space without any scale) to rather local viscosities. Such viscosities reflect more details of the geometrical conditions and of the time scales involved.

\section{Symmetry considerations}

The form (6) of the stress correlator follows directly from symmetry considerations. We will now apply the same considerations to derive the corresponding expression for a liquid close to a wall. Similar formulae are found in the literature on nematic liquids 15. The following symmetries apply to the stress correlator:

(A) The most rigorously enforced symmetry is that the stress tensor is symmetric. This symmetry is inherited from the microscopic definition of the stress tensor and is thus not subject to random fluctuations. It leads to the conditions

$$
\left\langle s_{i j} s_{k l}\right\rangle=\left\langle s_{j i} s_{k l}\right\rangle=\left\langle s_{i j} s_{l k}\right\rangle
$$

By this symmetry, the 81 components in the full correlation tensor of rank four in three-dimensional space are reduced to 36 different ones. 


\begin{tabular}{|c|c|c|}
\hline$\lambda+\frac{4}{3} \eta$ & $\begin{array}{c}\eta_{1} \\
\eta_{2}+\eta_{3}\end{array}$ & $\begin{array}{l}N N-N N \\
T T-T T\end{array}$ \\
\hline$\lambda-\frac{2}{3} \eta$ & $\begin{array}{c}\eta_{4} \\
\eta_{2}-\eta_{3}\end{array}$ & $\begin{array}{l}N N-T T \\
T T-T^{\prime} T^{\prime}\end{array}$ \\
\hline$\eta$ & & $\begin{array}{l}T T^{\prime}-T T^{\prime} \\
N T-N T\end{array}$ \\
\hline
\end{tabular}

Figure 1: Schematic breaking of viscosities in bulk fluid (left) into the surface viscosities near a flat wall (right). Given are the three/six non-zero values found in the components of the stress tensor correlator $A_{i j k l}$, indexed by the normal direction $N$ and the two tangential directions $T$ and $T^{\prime}$.

(B) Another symmetry is isotropy, that is the invariance of the correlator under rotations. It is not enforced by the microscopic definition and must result from the averaging either over an ensemble or possibly over a long trajectory. In bulk, the rotations are arbitrary, while in a geometry with plates one direction is distinct from the two others and only rotations parallel to the plates are permitted. The maximal number of different components is now reduced to 21 . They are, however, not all completely independent, and some of them vanish: A systematic decomposition of the rank-four tensor into parallel and orthogonal parts, using parallel isotropy and Eqs. (7), reveals that these 21 entries can take only nine different non-zero values, and that they depend on seven independent parameters (viscosities).

(C) The next symmetry is the invariance under index pair exchange,

$$
\left\langle s_{i j} s_{k l}\right\rangle=\left\langle s_{k l} s_{i j}\right\rangle .
$$

It is a consequence of the entropy production being a scalar 8]. This symmetry leads to the identification of two of the viscosities and the vanishing of another. After having used all symmetry requirements, we thus have a total of five viscosities left, which give rise to six different non-zero values in the 13 principally different components of the stress correlator.

The symmetry conditions near a plate require remarkably more viscosities than those in bulk, where two viscosities lead to three different values. The presence of the wall thus induces a separation of the bulk viscosities into surface viscosities. This splitting is depicted in Fig. 1. The diagram provides on the left-hand side the three (in bulk) different values found in the components of Eq. (6), and on the right-hand side the six non-zero values near a flat rigid immobile plane. The full rank-four tensor expressing the random stress correlations near a wall is

$$
\begin{aligned}
& A_{i j k l}=\eta_{1} N_{i} N_{j} N_{k} N_{l}+\eta_{2} g_{i j} g_{k l} \\
& +\eta_{3}\left(g_{i k} g_{j l}+g_{i l} g_{j k}-g_{i j} g_{k l}\right)+\eta_{4}\left(N_{i} N_{j} g_{k l}+N_{k} N_{l} g_{i j}\right) \\
& \quad+\eta_{5}\left(N_{i} N_{k} g_{j l}+N_{j} N_{k} g_{i l}+N_{i} N_{l} g_{k}+N_{j} N_{l} g_{i k}\right)
\end{aligned}
$$

The rightmost column of Fig. 1 uses a symbolic notation for the indices of tensor components. $N$ stands for the direction normal to the wall, $T$ may stand for both tangent directions, and $T^{\prime}$ is only used if both tangent directions are used: In particular, $T T$ stands for the two index combinations with the same tangent vector, whereas $T T^{\prime}$ stands for the ones with different tangent vectors. The same notation will be used in the figures of Sec. 4 to visualize the 13 components of the stress correlation tensor, six of which are non-zero and seven vanish.

\section{Coarse-graining of a microscopic stress tensor}

Let us consider a molecular dynamics simulation in order to study the microscopic dynamics of the molecules in a liquid. The liquid here consists of point-like atoms, the dynamics of which is governed by Hamilton's equations comprising a pair-interaction potential. In this dynamics we define a microscopic stress tensor. It is chosen such that its coarse-grained version, which is obtained by integrating it over a small region, will be consistent with a macroscopic notion of the hydrodynamic stress tensor.

The Hamiltonian of $N$ point-like atoms $(\alpha)$ of masses $m^{(\alpha)}$, positions $\mathbf{r}^{(\alpha)}(t)$ and linear momenta $\mathbf{p}^{(\alpha)}(t)$, which undergo mutual influence via a pair-potential $\phi$, reads

$$
H=\sum_{\alpha=1}^{N} \frac{\mathbf{p}^{(\alpha)} \cdot \mathbf{p}^{(\alpha)}}{2 m^{(\alpha)}}+\sum_{\alpha=1}^{N} \sum_{\beta=1}^{\alpha-1} \phi\left(\left\|\mathbf{r}^{(\beta)}-\mathbf{r}^{(\alpha)}\right\|\right) .
$$

The time evolutions of the positions and momenta are given by Hamilton's equations. The microscopic stress tensor $\tau_{i k}$, can then be introduced as the current density of the density of linear momentum $\mathbf{g}$, reading

$$
g_{i}(\mathbf{r}, t):=\sum_{\alpha} p_{i}^{(\alpha)}(t) \delta\left(\mathbf{r}-\mathbf{r}^{(\alpha)}(t)\right)
$$

In the absence of external forces, the conservation of momentum is expressed by the following balance equation, which introduces the microscopic stress tensor $\tau_{i k}$,

$$
\partial_{t} g_{i}(\mathbf{r}, t)-\partial_{r_{k}} \tau_{i k}(\mathbf{r}, t)=0
$$

The definition of these tensor fields allows us to pass from a description in terms of the atom positions $\mathbf{r}^{(\alpha)}(t)$ to a field description for any position $\mathbf{r}$. Equation 12 , which is at the same time a conservation theorem and a definition, fixes $\tau$ only partially. We may always add an arbitrary part with zero divergence, a gauge freedom which is inherent to the continuum description. However, we will not go into the implications of this freedom. A convenient definition of the microscopic stress tensor is the following, since it is symmetric and lends itself to a straight-forward coarse-

with the shortcut $g_{i j}:=\delta_{i j}-N_{i} N_{j}$. 
graining technique,

$$
\begin{aligned}
& \tau_{i k}(\mathbf{r}, t)=-\sum_{\alpha} \frac{p_{i}^{(\alpha)} p_{k}^{(\alpha)}}{m^{(\alpha)}} \delta\left(\mathbf{r}-\mathbf{r}^{(\alpha)}(t)\right) \\
&+ \frac{1}{2} \sum_{\alpha} \sum_{\beta \neq \alpha} \phi^{\prime}\left(\left\|\mathbf{r}^{(\beta)}-\mathbf{r}^{(\alpha)}\right\|\right) \frac{r_{i}^{(\alpha)}-r_{i}^{(\beta)}}{\left\|\mathbf{r}^{(\beta)}-\mathbf{r}^{(\alpha)}\right\|} \times \\
&\left(r_{k}^{(\alpha)}-r_{k}^{(\beta)}\right) \int_{0}^{1} \delta\left(\mathbf{r}-\mathbf{r}^{(\alpha)}-\lambda\left(\mathbf{r}^{(\beta)}-\mathbf{r}^{(\alpha)}\right)\right) d \lambda .
\end{aligned}
$$

The first term is usually called the kinetic part of the stress tensor. The second term comprises virial contributions, together with the integral of a three-dimensional delta function over the line starting at the point $\mathbf{r}^{(\alpha)}$ and ending at $\mathbf{r}^{(\beta)}$. Expression 13 is the same as that of Forster [16. see Eq. (4.6)], with the exception of the lower boundary of this integral.

We choose to do a coarse-graining by averaging the microscopic stress tensor within a small cube $V^{a}(\mathbf{x})$ of side-length $a$, centred around $\mathbf{x}$. We call this average the coarse-grained stress tensor $\tau_{i k}^{(a)}(\mathbf{x}, t)$ at the point $\mathbf{x}$,

$$
\tau_{i k}^{(a)}(\mathbf{x}, t):=\frac{1}{a^{3}} \int_{V^{a}(\mathbf{x})} \tau_{i k}(\mathbf{r}, t) d \mathbf{r} .
$$

The integration of the kinetic term in $\tau_{i k}$ is readily done. It uses the momenta of the particles which are found within the volume at time $t$. The line integral of the delta function has a simple geometrical interpretation. When integrated over the volume $V^{a}(\mathbf{x})$, only those parts of the line integral contribute where the line is found within the volume. For the (convex) cubic box $V^{a}(\mathbf{x})$, only four different cases are possible:

$$
\begin{gathered}
\left\|\mathbf{r}^{(\beta)}-\mathbf{r}^{(\alpha)}\right\| \int_{V} \int_{0}^{1} \delta\left(\mathbf{r}-\mathbf{r}^{(\alpha)}-\lambda\left(\mathbf{r}^{(\beta)}-\mathbf{r}^{(\alpha)}\right)\right) d \lambda d \mathbf{r} \\
=\left\{\begin{array}{cl}
\left\|\mathbf{r}^{(\beta)}-\mathbf{r}^{(\alpha)}\right\| & \text { if } \mathbf{r}^{(\alpha)} \in V \text { and } \mathbf{r}^{(\beta)} \in V, \\
\left\|\overline{\mathbf{r}}-\mathbf{r}^{(\alpha)}\right\| & \text { if } \mathbf{r}^{(\alpha)} \in V \text { and } \mathbf{r}^{(\beta)} \notin V, \\
\left\|\mathbf{r}^{(\beta)}-\overline{\mathbf{r}}\right\| & \text { if } \mathbf{r}^{(\alpha)} \notin V \text { and } \mathbf{r}^{(\beta)} \in V, \\
\left\|\overline{\mathbf{r}}-\overline{\mathbf{r}}^{\prime}\right\| & \text { if } \mathbf{r}^{(\alpha)} \notin V \text { and } \mathbf{r}^{(\beta)} \notin V .
\end{array}\right.
\end{gathered}
$$

Here, $\overline{\mathbf{r}}$ and $\overline{\mathbf{r}}^{\prime}$ denote the points where the connection line between the atoms intersect the boundary of the volume $V^{a}(\mathbf{x})$. The last case in Eq. 15 yields no contribution if these intersection points do not exist. Figure 2 provides a graphical representation of these different cases. For calculating the coarse-grained stress tensor we thus have to find the contributing atom pairs, to evaluate the pair potential, and to find the intersection points. Notice that even a box which does not contain any particle may yield a non-zero microscopic stress. It is sufficient that any connecting line passes through the box.

The usual way to calculate a stress tensor in molecular dynamics simulations is to add up the kinetic contribution and the full virial of the atoms. The simulation

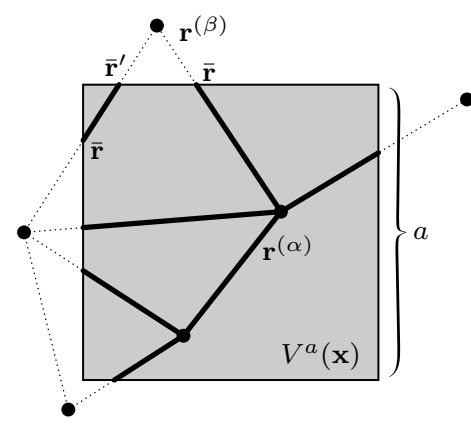

Figure 2: Visualisation of the virial contributions to the coarsegraining of the stress tensor. From the connection lines between the atoms (small circles) only the bold parts are taken into account. All cases of Eq. 15 are displayed.

package LAMMPS [17, for example, outputs the following quantity as stress per atom 1

$$
\begin{gathered}
\mathcal{T}_{i k}^{(\alpha)}:=-m^{(\alpha)} v_{i}^{(\alpha)} v_{k}^{(\alpha)}+\frac{1}{2} \sum_{\beta \in \text { Nbrs. }(\alpha)}\left(r_{k}^{(\alpha)}-r_{k}^{(\beta)}\right) \times \\
\frac{r_{i}^{(\alpha)}-r_{i}^{(\beta)}}{\left\|\mathbf{r}^{(\alpha)}-\mathbf{r}^{(\beta)}\right\|} \phi^{\prime}\left(\left\|\mathbf{r}^{(\alpha)}-\mathbf{r}^{(\beta)}\right\|\right) .
\end{gathered}
$$

However, $\mathcal{T}_{i k}^{(\alpha)}$ has the dimension of stress times volume. The stress is attributed to the atom with index $\alpha$, its neighbours have indices $\beta$. It is clear that $\mathcal{T}_{i k}^{(\alpha)}$ cannot be a local quantity, as it does not depend on any space variable. It may be the integral of a local quantity - such as the stress in Eq. 13) - over a specific volume around the atom $\alpha$-but over which volume? We find the answer to this question from the explicit averaging procedure above: Only if the particle and all its neighbours are within the volume of integration, that is the first case in Eq. (15), then we obtain expression (16) as the contribution coming from this atom. We conclude that $\mathcal{T}_{i k}^{(\alpha)}$ is the bulk contribution to a stress tensor when integrated over a volume which is sufficiently large that it contains the atom in question and all its interaction partners. It discards all boundary terms. For geometrical reasons it is not possible to assign space-filling individual volumes to all atoms (such as is done for example by a Voronoi tessellation) in order to obtain the stress per atom as an integral over the local stress. As the cell boundaries in the Voronoi tessellation are determined only by nearest neighbour's positions, they cannot correctly represent all interaction partner's positions, which are spanned over a larger region.

As our interest lies in the local fluctuations of the stress tensor, we have to keep the integration boxes as small as possible. The small boxes forbid the use of Eq. $(16)$, we have to average the microscopic stress tensor explicitly, according to the cases in Eq. 15. The additional work to be done in comparison with Eq. (16) is the solution of the geometrical intersection problem.

\footnotetext{
${ }^{1}$ Throughout the paper, terms specific to LAMMPS are typeset in a typewriter font.
} 


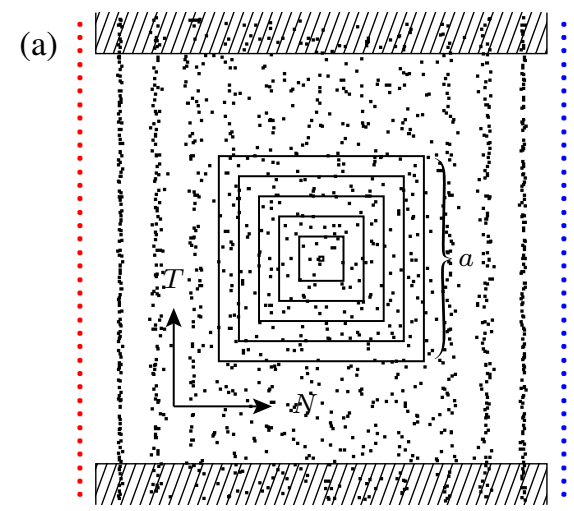

(b)

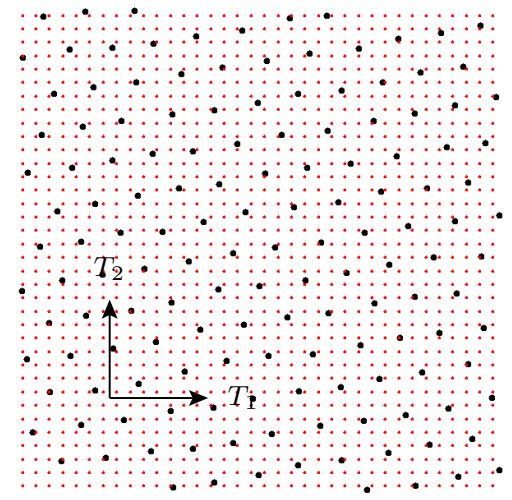

Figure 3: (Colour on-line) Snapshots of the simulation domain with $N=1380$ fluid atoms (black), confined between two fixed plates which consist of a regular grid of immobile atoms (red/blue). Shown are cuts through the domain as seen from two different sides. The series of cubes in the middle of panel (a) indicate the cubic boxes used for averaging in Sec. 4.2 In the hatched region, a Langevin thermostat controls the temperature.

\section{Results from molecular dynamics simulations}

The molecular dynamics simulations described in the following have been done with the molecular dynamics package LAMMPS [17. The coarse-graining procedure described above has been added as a module.

\subsection{Details of the simulation}

Our simulation is set up as a three-dimensional LennardJones liquid between two plates, as depicted in Fig. 3. 1380 fluid atoms are confined between two fixed plates. The plates are constructed as a single regular layer of atoms with the same interaction potential. In order to approximate a smooth flat surface, the lateral distance between these plate atoms is about a third of that in the fluid. The simulation domain is periodically continued in the two directions parallel to the plates.

The fluid atoms are advanced in time using the Verlet algorithm (NVE integration) which is consistent with the microcanonical ensemble. An exception are the hatched regions in Fig. 3 a, where an additional Langevin thermostat is applied. Such a thermostat is necessary to counteract numerical integration errors, but it modifies the statistics of the stress. We therefore chose to apply it only in regions where we do not measure the stress. The plate atoms are not advanced in time, simply by not applying any integration routine. The forces on these atoms are calculated, however, which is equivalent to the possible inclusion of strong constraining forces from crystalline layers behind. This procedure allows to determine the force exerted on the plates by the fluid.

The pair-interaction potential between the atoms is the 12/6 Lennard-Jones potential, modified by an interpolation term to have a finite carrier,

$$
\phi(r)=\left\{\begin{array}{cl}
4 \epsilon\left[(\sigma / r)^{12}-(\sigma / r)^{6}\right] & \text { for } r<2.5 \sigma \\
0 & \text { for } r>3.5 \sigma \\
\sum_{n=-3}^{2} \xi_{n}(\sigma / r)^{2 n} & \text { else. }
\end{array}\right.
$$

The six parameters $\xi_{n}$ are determined to guarantee continuous derivatives everywhere, up to the second. We use this smooth potential in order to make sure that discontinuities in the second derivative do not produce artefacts in the measurement of the stress tensor.

In the following, we will use Lennard-Jones units for all quantities. In these units, distances are measured as multiples of the length parameter $\sigma$ in the Lennard--Jones potential, energy as multiples of $\epsilon$, and time in terms of $\sqrt{m \sigma^{2} / \epsilon}$, where $m$ is the mass unit. In these units, the mass of the atoms is chosen to be 1 , the side-length of the simulation domain is fixed to be 12 . The time unit is approximately the oscillation period in the minimum of the pair potential $\phi$. The time-step of integration, used in the Verlet algorithm is 0.002 , thus more than two orders of magnitude smaller than all physical time scales in the system.

The density and the (kinetic) temperature of the system are chosen such that it is found in its liquid state, according to the phase diagram in Refs. [18, 19, 20]. Of course, this holds only in the "bulk" region where the structuring effect of the walls is absent. The disordered fluid is visible in the middle of Fig. 3 a. There, the following average values for temperature, density and pressure are found: $T=1.04, \rho_{0}=0.83, p_{0}=1.3$. Near the plates, the enforced flat geometry imposes a partial ordering of the fluid. Up to three distinct layers of fluid atoms can be identified near each wall. The first layer has a locally hexagonal structure as seen in Fig. 3 p. Its width and orientation are independent of the grid spacing of the plate atoms. Due to periodic boundary conditions, the hexagonal grid does not rotate anymore as soon as the system is equilibrated. Of course, also the stress tensor becomes anisotropic close to the plates.

The simulation was first run to let the system find its thermodynamic equilibrium. We then continued the simulation while calculating the coarse-grained stress tensors at some fixed positions every 5 time steps. These time series are analysed in the following, either using the blocking method 21 to ensure that all used variables are equilibrated, or by simple averaging to measure spatial correlations of the stress tensor, or by fast Fourier transforms for 


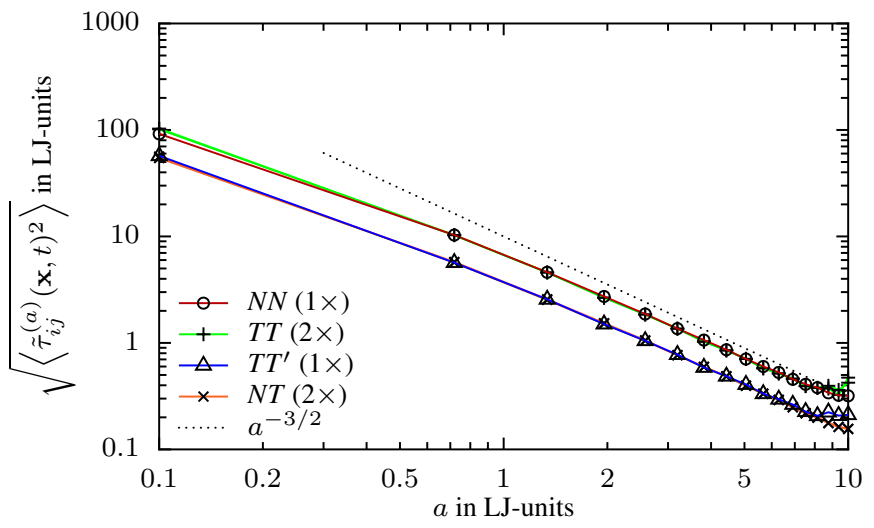

Figure 4: (Colour on-line) Fluctuations of the stress tensor in bulk, as a function of the side $a$ of the averaging box. All tensor components are plotted. They are grouped according to the expected symmetry (see text).

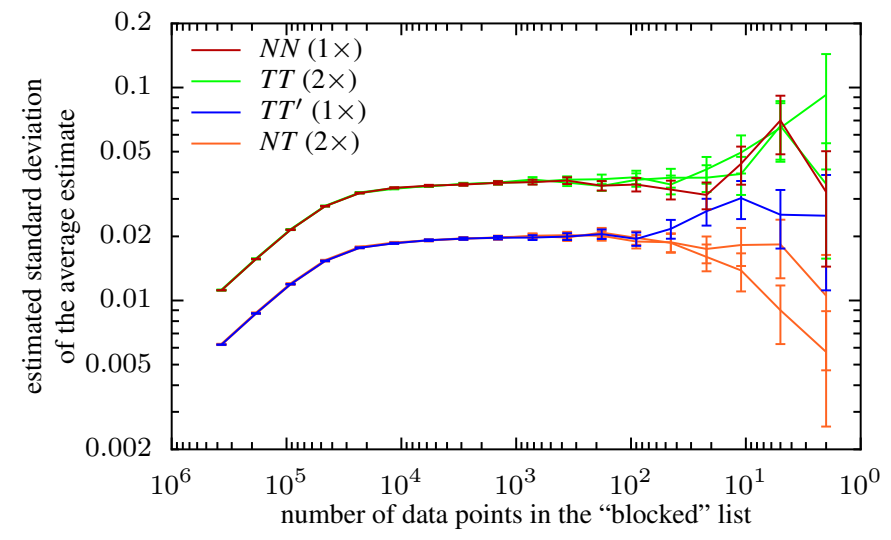

Figure 5: (Colour on-line) Estimated error of the average estimation from the blocking transformation [21. We used the time series of all stress components in a box of size $a=1.0$ centred between the plates.

the time correlations. The averages $\langle\cdot\rangle$ in the following are thus time-averages over a long trajectory. As the system is in equilibrium, this should be equivalent to an ensemble average.

\subsection{Varying the size of the coarse-graining boxes}

Before presenting the temporal and spatial dependence of the stress correlator, let us investigate how the side length $a$ of the averaging box changes the fluctuations of the coarse-grained stress tensor inside.

The explicit coarse-graining method by averaging over small cubes allows to observe the transition from thermodynamic behaviour (in large boxes) to highly fluctuating random behaviour (in small boxes). The amount of fluctuations depends of course on the size of the box. As we treat here only thermodynamically equilibrated fluids, the mean square deviation of the stress tensor should scale as the inverse volume of the averaging box - just as for any intensive quantity [22, $\S 112]$. In the bulk fluid, we should thus find the deviations of the stress tensor to be

$$
\sqrt{\left\langle\tilde{\tau}_{i j}^{(a)}(\mathbf{x}, t)^{2}\right\rangle} \propto a^{-3 / 2}
$$

where $\tilde{\tau}_{i j}^{(a)}(\mathbf{x}, t)$ is defined by subtracting the macroscopic stress tensor, which is $-p_{0} \delta_{i j}$,

$$
\tilde{\tau}_{i j}^{(a)}(\mathbf{x}, t):=\tau_{i j}^{(a)}(\mathbf{x}, t)+p_{0} \delta_{i j}
$$

The value for the pressure is taken from disordered fluid atoms only, and not from the layers close to the plates.

The numerical verification of the scaling $(18)$ is plotted in Fig.4. Amazingly, we find the correct scaling already for box sizes of $a \sim 2$, that is a side length of the order of the average distance between the fluid atoms. The scaling (18) is originally based on a thermodynamic argument which requires that many particles are involved. Instead, we here see the correct scaling already for very few particles. It appears that there is more truth in the thermodynamic argument than just the interaction of many particles.

For visualising the tensor of stress fluctuations, we have chosen the following strategy, which will be applied also to the following plots: The symmetry requirements (B) and (C) in Sec. 2 lead to components which are equal only in a statistical sense. All components which may exhibit such fluctuations are plotted by individual lines. The legend and the colour coding, however, differentiate only between the groups of components which should be equal on average. The number of lines actually plotted is given in parentheses.

The fact that there is no difference between the normal and the parallel directions in the data of Fig. 4 confirms our assumption that the fluid behaviour in the middle between the walls is the same as if they were absent. There, the diagonal and the off-diagonal parts fluctuate differently. This is a consequence of the components being different functions of the two viscosities (see also Fig. 1).

Exemplary for all plotted variables, we show for the centred boxes that the system is well equilibrated. Fig. 5 visualises the blocking transformation [21] of the diagonal entries in the coarse-grained stress tensor. A plateau is clearly visible, which shows that the analysed time series indeed correspond to a stationary stochastic process.

\subsection{Stress correlations in bulk fluid}

The aim of this paper is to determine the spatial and the temporal structure in correlations of fluctuations of the coarse-grained stress tensor $\tilde{\tau}_{i k}^{(a)}$. We are interested in the difference between correlations in bulk and in the near vicinity of the walls. In order to do so, we set out three different series of cubic boxes, as depicted in Fig. 6. These cubes are shifted by small distances and may overlap. The first series is in the middle between the plates, where the fluid behaves as in bulk, a second one close to a wall and parallel to it, and a third series is normal to the wall.

The displacement of the boxes makes it possible to determine the spatial form of the stress correlation by 


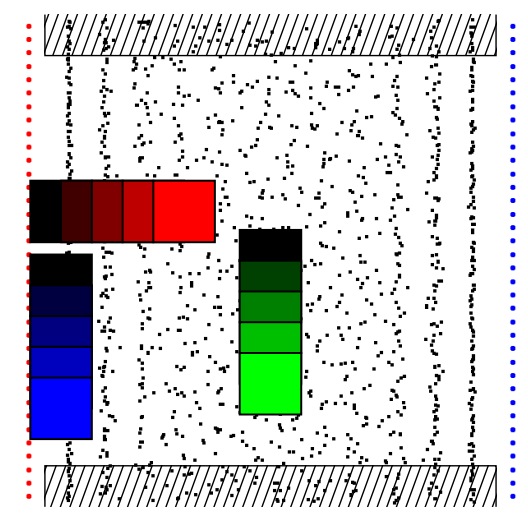

Figure 6: (Colour on-line) Same as Fig. 3a, but with different series of overlapping coarse-graining cubes, which are used in Figs. 7] 10

cross-correlating the coarse-grained stress fluctuations in different boxes. Temporal correlation functions will also be given. We would like to compare the result with the uncorrelated theory in Eq. (5). However, the nonzero size of the boxes used for averaging does not allow a direct comparison. Remind that $\tilde{\tau}_{i j}^{(a)}$ depends on the box size. We should find the delta function of Eq. (5) in the limit $a \rightarrow 0$, which is infeasible in practice. For all nonzero sizes, the numerical data is sort of "convoluted" with the shape of the boxes. The best we can do is to compare with the equally smoothed version of Eq. (5) using a reasonably small $a$. The corresponding correlator of the uncorrelated stress tensor $s_{i j}$ is expressed by the relative overlap of the two boxes,

$$
\left\langle\tilde{\tau}_{i j}^{(a)} \tilde{\tau}_{k l}^{(a)}\right\rangle \propto\left|V^{a}(\mathbf{x}) \cup V^{a}(\mathbf{y})\right|
$$

of course disregarding the infinite term $\delta(t-s)$ in Eq. (5). Here, $|\cdot|$ denotes taking the volume of a set.

The spatial and the temporal correlations of the random coarse-grained stress tensor are depicted in Fig. 7 . The data have been generated by correlating the contents each box in the central series of Fig. 6 with the first one, which has $\mathbf{x}_{0}$ as its centre. Plotted are all 36 components of the resulting rank-four tensor which can be different using the symmetry (A) of Sec. 2. They are grouped into the 13 different classes according to the symmetries (B) and (C). Fig. 7 shows that seven of them vanish (grey lines) and that the remaining six take three different values. This is expected according to what has been said around Fig. 1 .

The spatial correlations in Fig. 7 a should be compared with the results expected from an uncorrelated stress tensor. These would be lines starting at some value which is related to the viscosities (unspecified due to $\delta(t-s)$ in Eq. (5p) and which goes to zero the distance $a$ and beyond. The coincidence in Fig. $7 \mathrm{a}$ is remarkable. The numeric values are a bit more smoothed, but the apparent convolution kernel responsible for this smoothing has a width of less than unity. We therefore conclude that Eqs. (5) with Eq. (6) in bulk are very precise, down to a length scale of the order of the molecule distance. Similarly to what we found in Sec. 4.2, such a strong result cannot be expected from thermodynamic considerations.

The temporal correlations in Fig. $7 \mathrm{p}$ exhibit the deviations from the temporal delta function in Eq. (5). The decay is sufficiently fast to reasonably adopt the approximation of the delta function. Whether there is an algebraic contribution, the so-called long-time tail cannot be judged from these data. In order to calculate the viscosity values, we use the integral over the detailed time correlator, as proposed by the Green-Kubo equation. The idea is the same as in Refs. [23, 24, 25, only that we take a box smaller than the simulation box. For the shear viscosity, this reads

$$
\eta=\frac{a^{3}}{k T} \int_{0}^{\infty} d t\left\langle\tilde{\tau}_{N T}^{(a)}(\mathbf{x}, 0) \tilde{\tau}_{N T}^{(a)}(\mathbf{x}, t)\right\rangle,
$$

and similarly for the two components comprising the volume viscosity $\lambda$. From the data in Fig. $7 \mathrm{p}$ we find the values $\eta=0.84$ and $\lambda=1.72$ by regression. 2

\subsection{Stress correlations near a rigid flat wall}

Near the plates, the stress correlations look quantitatively and qualitatively different. The average stress tensor and also its fluctuations are anisotropic. Our aim in this section is to determine the five surface viscosities in order to quantify their deviations from the bulk values, according to the scheme in Fig. 1.

First, let us look at the coarse-grained random stress tensor itself. Fig. 8 reveals the mechanical properties of the first fluid layers: We find a huge lateral diagonal stress (TT components) which strongly depends on the distance to the wall but not on the lateral position. In the figure, the points $\mathbf{x}$ correspond to the centres of the series of boxes laid out in Fig. 6. The position $\mathbf{x}_{0}$ is the centre of the first box in the normal series, which is closest to the wall. The average value of the huge diagonal stress is even larger than its fluctuations. This lateral stress can be understood as a surface tension of the contact between liquid and solid. The $N N$-component is much smaller-its average value is the same as the bulk pressure. The $N N$ component is dominated by its fluctuations which grow in the vicinity of the wall and even overgrow the tangential stresses. In the following, we will find that there are correlations in these fluctuations. The lines in Fig. $8 \mathrm{~b}$ prove that both the average values and the standard deviations are independent of the lateral position. The roughness of the $N N$-component in this plot is due to the large fluctuations.

\footnotetext{
${ }^{2}$ Notice that these values do not quite correspond to the finding in Refs. 24, 25]. A possible explanation is the dependence of the viscosities on the size $a$ of the averaging box. We found such a dependence together with long-time tails in the autocorrelation of the random stress tensor in bulk.
} 

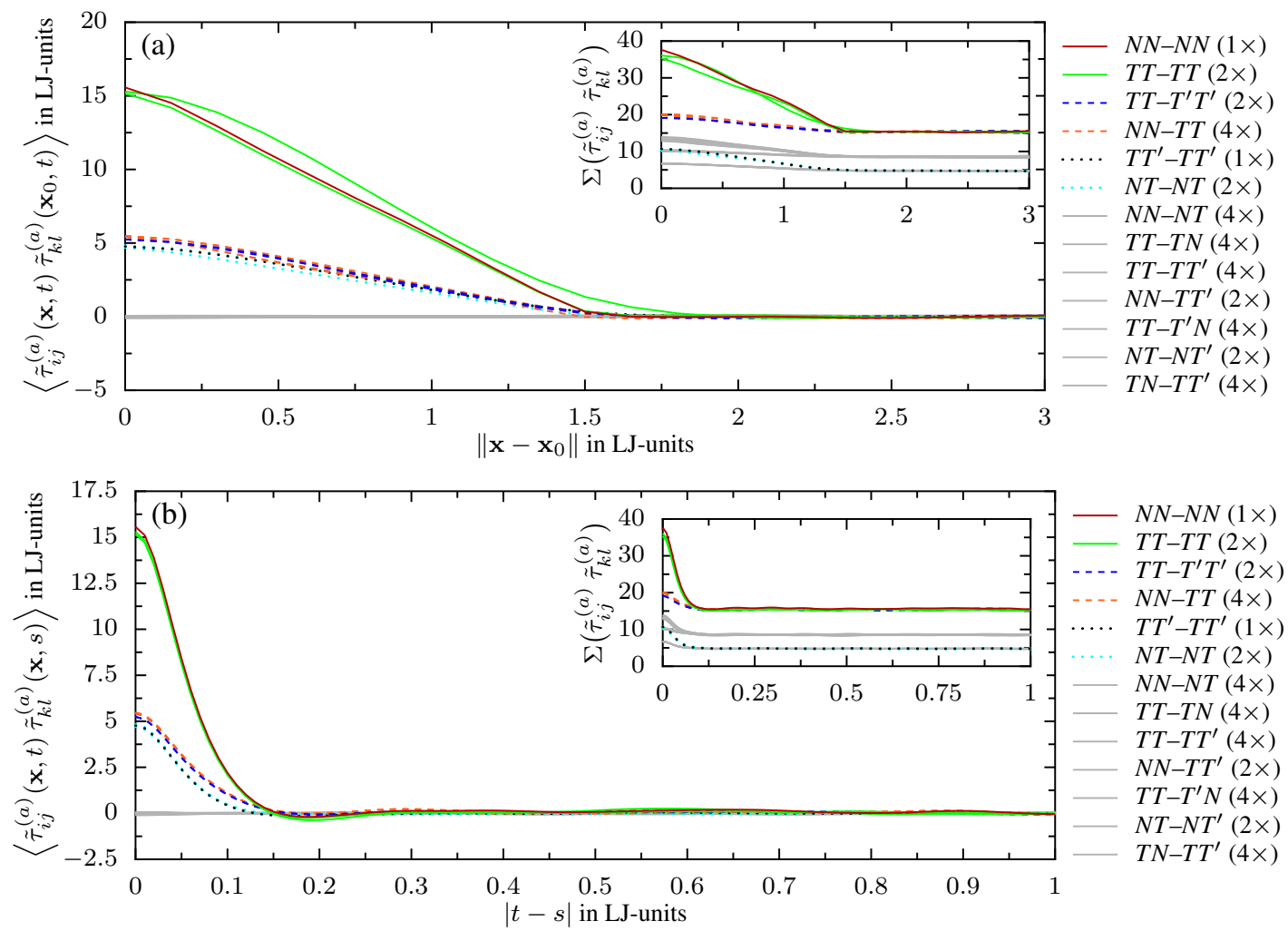

Figure 7: (Colour on-line) Spatial and temporal correlations of the stress fluctuations in the middle of the domain (in bulk). Plotted are the average correlators and their standard deviations $(\Sigma(\cdot)$, see insets) for different classes of index combinations.

For the data in Fig. 8 and in the following ones, another comment on the symmetry is necessary. By the noted symmetries in Sec. 2 the two lines for the TT-components in Fig. 8 should coincide. However, they do not. The reason is that the structured liquid is not entirely isotropic in the tangential directions, but it is ordered in a hexagonal grid, as depicted in Fig. 3b. This strict ordering is a result of the wall being very flat. A roughness of the size of one atom distance would easily destroy the hexagonal structure, and only the symmetries of Sec. 2 would remain. In order to weaken the effect, we averaged over eight trajectories which differed only by the orientation of the hexagonal grid. This reduced the deviations considerably to those in Fig. 8 .

The spatial correlations of the random stress tensor are depicted in Fig. 9. Again, the two series of integration boxes in different directions are used, see Fig. 6. The plot shows the correlation of each cube in the series with the first one, which is in the normal series the one closely aligned at the wall. For the normal series, the result is not expected to be independent of this choice. Only in the parallel series, the correlation should depend only on the distance of the boxes. As explained above, there are in principle 13 different results to be expected. Seven of them, those indicated in grey, are zero. The six non-zero components are plotted in colour. The correlations of the stress tensor inherit the main features of the stress in Fig. 8, in particular the systematic part which we called a surface tension. In normal direction, the tangential components exhibit a similar anti-correlation as in Fig. 8b. The decay of the most pronounced correlations takes place on the length of one atom-distance and is independent of the box size $a$. We verified this statement using other box sizes. In tangential direction, the surface tension manifests itself in a nonzero value of the tangential correlation, see Fig. 8 p.

Apart from the surface tension, we find in Fig. $8 \mathrm{~b}$ a similar behaviour as in bulk, that is a linear decay up to a distance $a$ and zero beyond. This is clearly visible for the $N N-N N$ component, but also for the others - after subtracting the contribution of the surface tension. This implies that the spatial delta function still works parallel to the walls. The correlation in normal direction, which is dominated by the details of the interaction between wall and fluid, depends both on the distance between the box and their distance to the wall. These two considerations lead us to the following proposition for the correlation function of the random stress tensor near an immobile flat rigid wall:

$$
\begin{aligned}
&\left\langle s_{i j}(\mathbf{x}, t) s_{k l}(\mathbf{y}, s)\right\rangle=2 k T A_{i j k l} \delta(t-s) \times \\
& \delta^{2}\left(\mathbf{x}^{\|}-\mathbf{y}^{\|}\right) f\left(\mathbf{x}^{\perp}, \mathbf{y}^{\perp}\right) .
\end{aligned}
$$

with the tensor $A_{i j k l}$ from Eq. (9), depending on the surface viscosities, and with $\mathbf{x}^{\|}$and $\mathbf{x}^{\perp}$ the parallel and normal projections, respectively. The function $f$, which has the physical dimension of inverse length, takes into ac- 

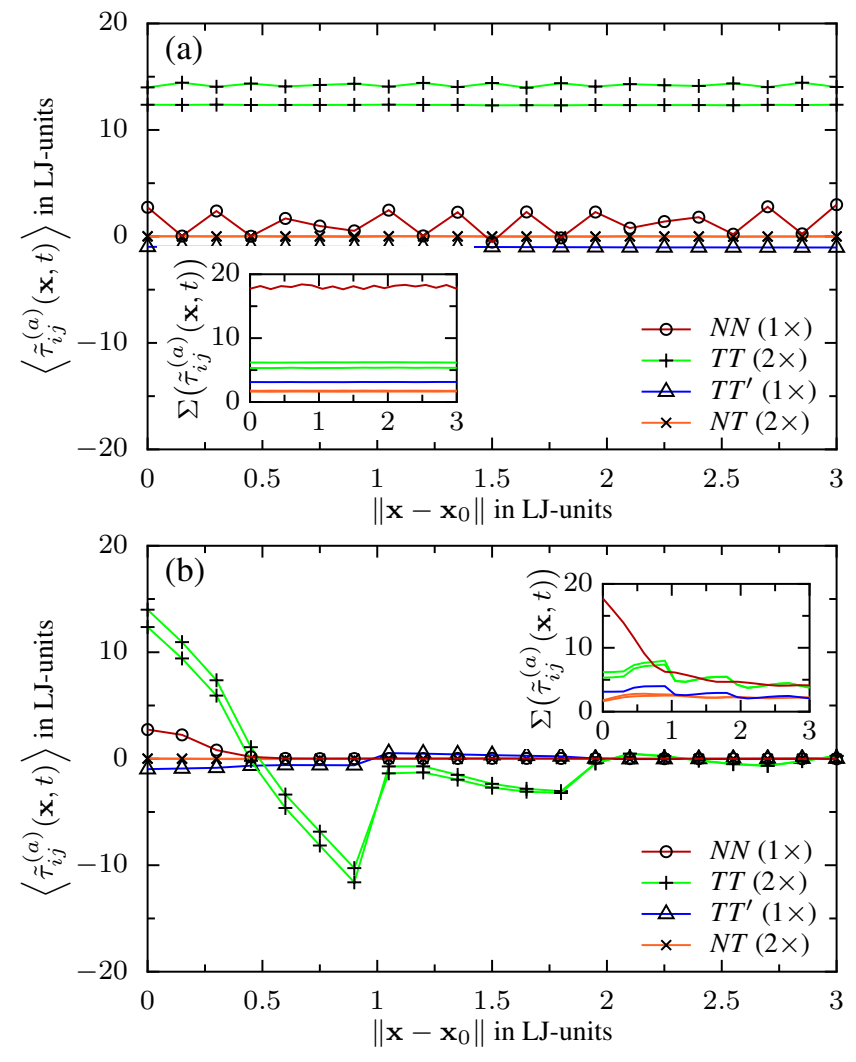

Figure 8: (Colour on-line) The coarse-grained stress tensor as a function of the position, as indicated in two series close to the wall in Fig. 6 (a) parallel to the plate, (b) orthogonal to the plate.

count the microscopic details of the interaction between wall and fluid. As the only length scale involved is the distance between wall and the first structured liquid layer, we expected $f$ to scale as one over this distance.

It remains to check whether the delta function in time is still a good approximation, and what values the surface viscosities have. The temporal correlations near the wall are depicted in Fig. 10. The results are taken from one of the boxes in the parallel series. Here, we see even better than in Fig. 9 that there is a systematic contribution coming from the surface tension. Notice that it affects only the tangential components and not the heavily oscillating $N N-N N$ component, which is decaying to zero, but on a longer time scale than plotted.

In order to obtain the viscosities from these data, we have subtracted the asymptotic value from the correlation function, which renders the function integrable. This means that we implicitly subtracted a sort of local pressure which is allowed to be anisotropic. This pressure reflects the static contributions from the interaction liquid-wall, which we called surface tension above. The Green-Kubo integral of the such normalized correlation functions yields the following values for the surface viscosities:

$$
\begin{aligned}
& \eta_{1} \approx 113, \\
& \eta_{2} \text { between } 16 \text { and } 34, \\
& \eta_{3} \approx 8.4 \\
& \eta_{4} \text { between } 65 \text { and } 178, \\
& \eta_{5} \text { between } 4.2 \text { and } 5.5 .
\end{aligned}
$$

The values for these viscosities are not very precise for three reasons: The first is the hexagonal structure of the first fluid layer which does not coincide with the Cartesian coordinates used for the tensor indices. We thus expect different viscosity values for different orientations of the hexagonal grid. The above mentioned average over eight different trajectories with different orientations apparently does not suffice to obtain unified values. The second reason is that the thermodynamic pressure is not isotropic anymore and that the time-correlation function does not decay to zero. We thus had to strip off a linear timedependency before integrating, as already explained. The third reason is that the intrinsic time scales of the timecorrelation function are much longer than in bulk. While the bulk viscosities could be easily identified from a GreenKubo integral over a few dozens of timesteps, it required thousand and more timesteps to do the same for the surface viscosities. This much longer time scale can already be guessed from the comparison of Figs. $7 \mathrm{~b}$ and 10 .

\subsection{Hydrophobic walls}

The rigid plates used in the above simulations consist of the same type of atoms as the liquid. Such walls are known to be hydrophilic, which is also seen in the strict ordering of the first structured fluid layer. We repeated the simulation using hydrophobic walls, which have a modified LennardJones potential to interact with the fluid atoms [26],

$$
\phi(r)=4 \epsilon\left[(\sigma / r)^{12}-c(\sigma / r)^{6}\right]
$$

with the same cutoff and with an equivalent smoothing of the potential as in Eq. (17). According to Barrat and Bocquet [26], who proposed the form (24) for simulating hydrophobic walls, the factor factor $c=0.5$ which we used, should correspond to a contact angle of about $140^{\circ}$.

The simulation leads to a similarly structured liquid as in Fig. (3), only that the first layers are a bit farther away from the walls, that they are not as flat, and that their lateral structure is not as regular. The ordering effect of the first layers are nevertheless very strong, and must therefore be induced by the flat geometry of the wall. We took the same bulk pressure and density as in the previous simulation. As expected for a hydrophobic surface, the TTcomponents in Fig. 8 are different. They are considerably smaller, around the value 3 instead of 13 . The correlation functions look similar to those depicted in Figs. 9 and 10. with the difference that they decay much faster in time, in particular the $N N-N N$ component. Accordingly, also 

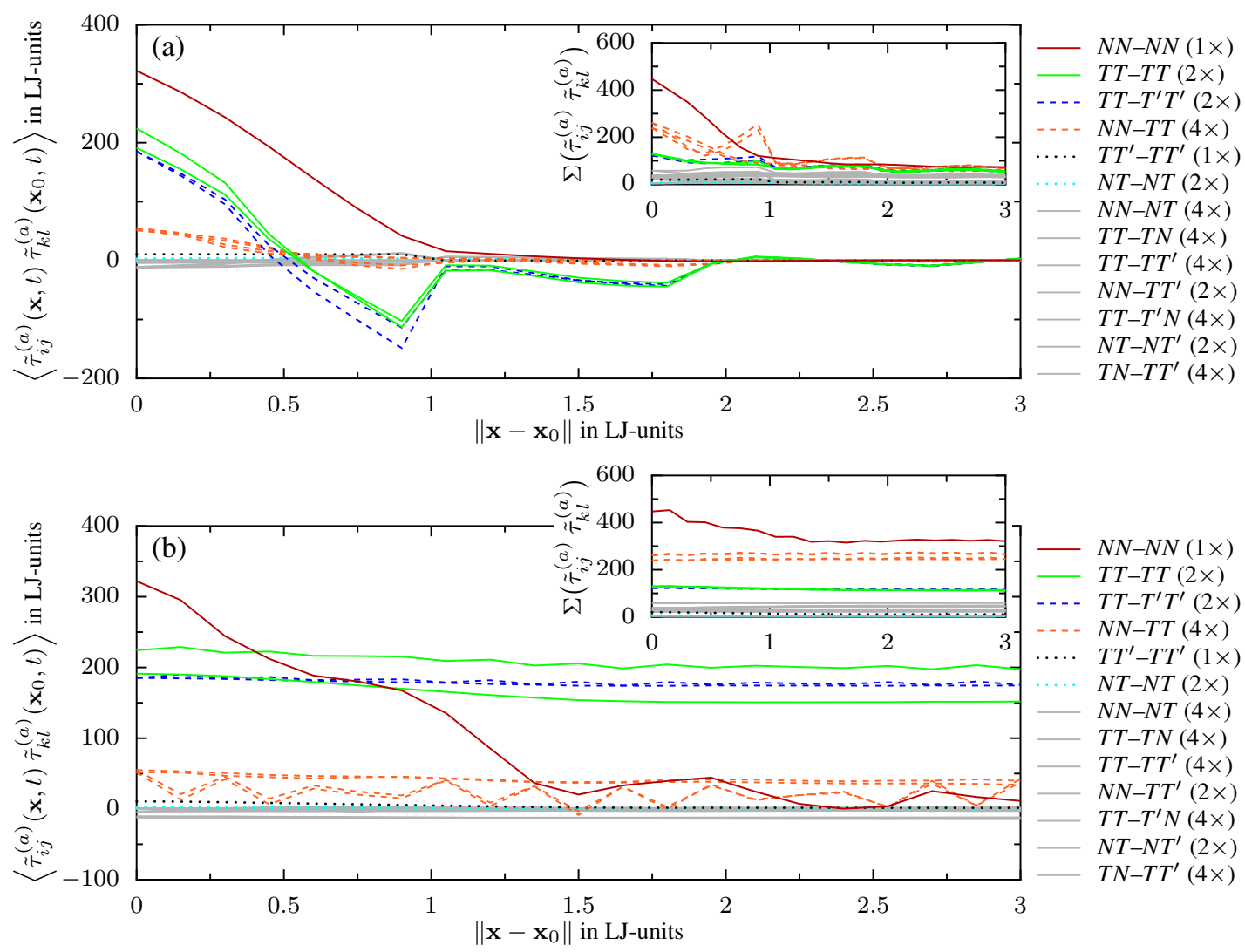

Figure 9: (Colour on-line) Spatial correlations of the random stress tensor in the vicinity of a wall. (a) in normal direction, and (b) parallel to the wall, see the series of cubes in Fig. 6. Each box in the series is correlated with the first one, centred around $\mathbf{x}_{0}$ (in the orthogonal case: the one closest to the wall). Legend and inset as in Fig. 7 .

the surface viscosities turn out to be different. Near the hydrophobic surfaces, we find the values

$$
\begin{aligned}
\eta_{1} & \approx 87, \\
\eta_{2} & \approx 1.7, \\
\eta_{3} & \approx 1.5, \\
\eta_{4} & \approx 8.1, \\
\eta_{5} & \approx 0.35 .
\end{aligned}
$$

It is not astonishing that these viscosities are smaller than those found near the wetting walls. In particular in tangential direction, where the liquid is feels less constraints, the dissipation is smaller. This affects $\eta_{2}-\eta_{5}$, but not $\eta_{1}$, which is accordingly of a similar magnitude as in the wetting-wall case. Particularly astonishing is the small value of $\eta_{5}$, which takes over the role of the shear viscosity. It is much smaller than the bulk value $(\eta=0.84$ also in the hydrophobic simulation).

\section{Discussion}

The numerical observations above, as described in Sections 4.3 and 4.4 allow to estimate the validity of the main assumptions leading to fluctuating hydrodynamics. Indeed, we observed that the spatial delta function in Eq. (5) works very well in bulk. Near a plate, which has been assumed to be flat, immobile and rigid, the isotropy is restricted to rotations parallel to the wall, and we must replace the spatial delta function by one which is only acting parallel to the wall, as given in Eq. 222. In orthogonal direction, the details of the dynamical interaction between liquid and wall introduce an unknown factor in form of a function depending on the orthogonal distances. Moreover, we have to deal with five viscosities instead of two, the values of which are provided in Eq. (23).

The parallel structure of the spatial delta functions and the number of viscosities are both imposed by pure symmetry and cannot be disputed. But we may ask which of the surface viscosities will have an effect on measurable quantities, such as on the flow profile in nanopores or on the motion of a Brownian particle. If we regard only forces exerted on the rigid walls - and their correlation functions-, there are only two viscosities which may play a role, namely those possessing a normal direction in both contributing stresses. They are easily identified in the scheme in Fig. 1 to be $\eta_{1}$ and $\eta_{5}$, the latter of which takes over the role of the bulk shear viscosity, and the first one the role of the bulk volume viscosity. It is interesting to see that the value of $\eta_{5}$ is entirely independent on the shear viscosity in bulk. It depends more on the hydrophobic/hydrophilic character of the walls. The 


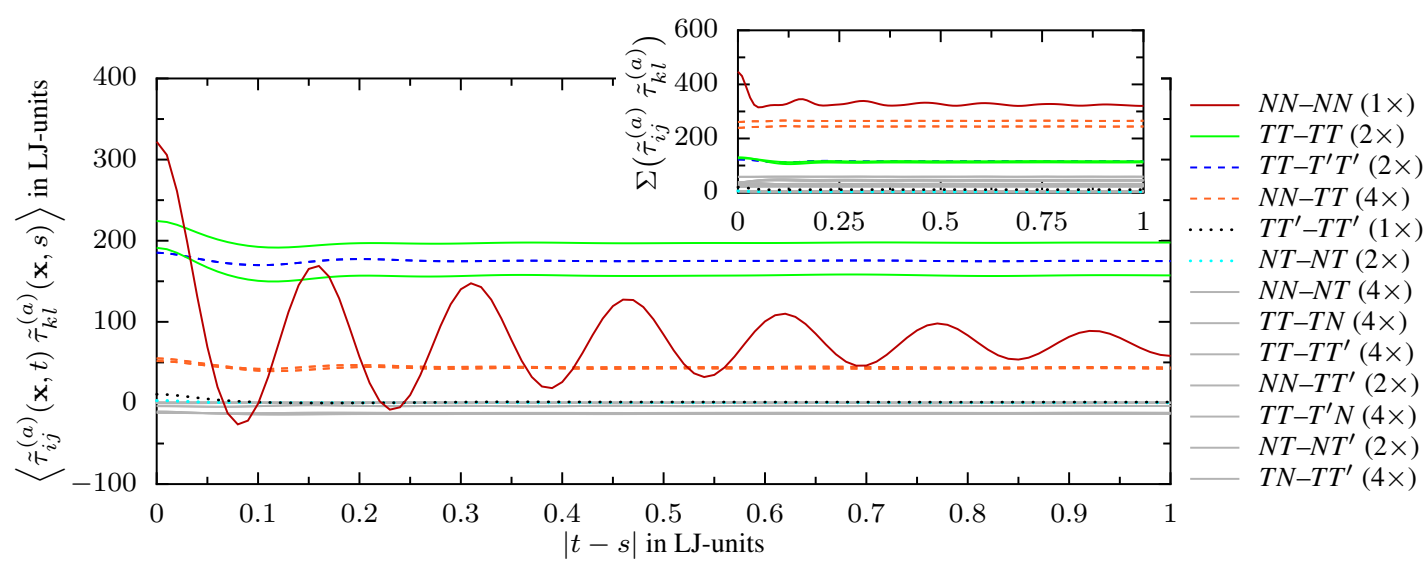

Figure 10: (Colour on-line) Temporal correlations of the random stress tensor near a wall. Legend and inset as in Fig. $7 \mathrm{~b}$.

surface-equivalent of the volume viscosity, $\eta_{1}$, is in both cases nearly two orders of magnitude larger than in bulk.

The finding that the surface viscosities do not have values comparable to the bulk viscosities brings us now to more profound questions on the dynamical theory involved: For the Brownian particle, we argue that only the first surrounding liquid layers do actually interact with the particle. There, the relevant dissipation coefficients are not the bulk viscosities but the two viscosities $\eta_{1}$ and $\eta_{5}$. It appears thus as a reasonable to ask why we do not see $\eta_{5}$ instead of $\eta$ appearing in the Stokes-Einstein formula for the diffusion coefficient? We have to leave this question open at the moment. A possible answer might go in the same direction as the discussion of time correlations and spatial ordering effects of Ref. [27].

A possible answer is certainly linked to the fact that the surface viscosities here are local quantities. They have been determined using coarse-graining over very small boxes. The usual definition of a "viscosity", however, implies limits to large boxes and long time integrals (or the limit $\omega \rightarrow 0$ after the limit $\mathbf{k} \rightarrow \mathbf{0}$ in Fourier space and time [3. p. 186]). In this limit, the five surface viscosities cannot be identified anymore. Another implication of the use of $l o-$ cal viscosities, which are not viscosities in the usual sense of a large-box limit, is that they do not lead to differential equations. They rather appear as integral kernels, nonlocal both in space and in time, in integro-differential equation. We cannot say much about the possibility to find an to interpret such equations at the moment, the less as we expect the long-time tails in the autocorrelation function, thus nonlinear terms, to intervene. Nevertheless, we would like to stress that we do not expect the qualitative nature of the anisotropy described here - that is the large difference of surface and bulk viscosity values, and that the surface viscosities do not depend on the bulk viscosities - to depend on the precise temporal behaviour. The long-time tails appearing in the correlation functions should only change the quantitative values of the viscosities, leaving the presented material untouched.

\section{Acknowledgements}

I would like to dedicate this paper to Peter Hänggi, on occasion of his 60th birthday, which is celebrated by the present special issue of Chemical Physics. The subject of this paper, in particular the aspects explained in the last section touches in part his early works on stochastic processes with memory.

I would further like to express my thanks to Peter Talkner for waking my interest in fluctuating hydrodynamics, and to Laurent Joly and Lydéric Bocquet for introducing me to the molecular dynamics package LAMMPS. Further sincere thanks go to Anthony C. Maggs for pointing me to the blocking method and to Claire Lemarchand, Ken Sekimoto, Falko Ziebert and Jörg Rottler for helpful discussions.

\section{References}

[1] L. D. Landau, E. M. Lifshitz, Fluid Mechanics, Pergamon Press, 1959.

[2] R. F. Fox, G. E. Uhlenbeck, Contributions to Non-Equilibrium Thermodynamics. I. Theory of Hydrodynamical Fluctuations, 197013 (Phys. Fluids.) 1893.

[3] R. Kubo, M. Toda, N. Hashitume, Statistical Physics II. Nonequilibrium Statistical Mechanics, Springer Verlag, 1985.

[4] H. Grabert, P. Talkner, P. Hänggi, Microdynamics and TimeEvolution of Macroscopic Non-Markovian Systems, Z. Physik B 26 (1977) 389.

[5] E. H. Hauge, A. Martin-Löf, Fluctuating hydrodynamics and Brownian motion, J. Stat. Phys. 7 (1973) 259.

[6] T. S. Chow, J. J. Hermans, Effect of Inertia on the Brownian Motion of Rigid Particles in a Viscous Fluid, J. Chem. Phys. 56 (1972) 3150 .

[7] G. Temple, Cartesian Tensors. An Introduction, Methuen, London, 1960.

[8] S. R. de Groot, P. Mazur, Non-Equilibrium Thermodynamics, Dover Publications, New York, 1984.

[9] C. Kettner, P. Reimann, P. Hänggi, F. Müller, Drift Ratchet, Phys. Rev. E 61 (2000) 312.

[10] Z. Guttenberg, A. Rathgeber, S. Keller, J. O. Rädler, A. Wixforth, M. Kostur, M. Schindler, P. Talkner, Flow profiling of a surface-acoustic-wave nanopump, Phys. Rev. E 70 (2004) 056311 . 
[11] M. Schindler, P. Talkner, M. Kostur, P. Hänggi, Accumulating particles at the boundaries of a laminar flow, Physica A 385 (2007) 46.

[12] K. John, P. Hänggi, U. Thiele, Ratched-driven fluid transport in bounded two-layer films of immiscible liquids, Soft Matter 4 (2008) 1183.

[13] L. Bocquet, E. Charlaix, Nanofluidics, from bulk to interfaces, Chem. Soc. Rev. 39 (2010) 1073.

[14] M. Schindler, Boundary-integral formulation of particle motion in unsteady Stokes/Oseen flow, submitted .

[15] S. Bohlius, H. R. Brand, H. Pleiner, Macroscopic dynamics of uniaxial magnetic gels, Phys. Rev. E 70 (2004) 061411.

[16] D. Forster, Hydrodynamic fluctuations, broken symmetry, and correlation functions, Addison-Wesley, 1990.

[17] S. J. Plimpton, Fast Parallel Algorithms for Short-Range Molecular Dynamics, J. Comp. Phys. 117 (1995) 1.

[18] B. Smit, Phase diagrams of Lennard-Jones fluids, J. Chem. Phys. 96 (1992) 8639.

[19] M. A. van der Hoef, Free energy of the Lennard-Jones solid, J. Chem. Phys. 113 (2000) 8142.

[20] E. A. Mastny, J. J. de Pablo, Melting line of the LennardJones system, infinite size, and full potential, J. Chem. Phys. 127 (2007) 104504.

[21] H. Flyvbjerg, H. G. Petersen, Error estimates on averages of correlated data, J. Chem. Phys. 91 (1989) 461.

[22] L. D. Landau, E. M. Lifshitz, Statistical Physics, Part 1, Butterworth-Heinemann, Oxford, 3rd edn., 2001.

[23] R. L. Rowley, M. M. Painter, Diffusion and Viscosity Equations of State for a Lennard-Jones fluid Obtained from Molecular Dynamics Simulations, Int. J. Thermophys. 18 (1997) 1109.

[24] K. Meier, A. Laesecke, S. Kabelac, Transport coefficients of the Lennard-Jones model fluid. I. Viscosity, J. Chem. Phys. 121 (2004) 3671.

[25] K. Meier, A. Laesecke, S. Kabelac, Transport coefficients of the Lennard-Jones model fluid. III. Bulk viscosity, J. Chem. Phys. 122 (2005) 014513.

[26] J.-L. Barrat, L. Bocquet, Large Slip Effect at a Nonwetting Fluid-Solid Interface, Phys. Rev. Lett. 82 (1999) 4671.

[27] A. V. Mokshin, R. M. Yulmetyev, P. Hänggi, Diffusion processes and memory effects, New J. Phys. 7 (2005) 9. 